\title{
Recent progress in combinatorial random matrix theory
}

\author{
Van H. Vu \\ Abstract: We are going to discuss recent progress on many problems in \\ random matrix theory of a combinatorial nature, including several break- \\ throughs that solve long standing famous conjectures. \\ Received May 2020.
}

\section{Introduction}

The theory of random matrices is a very rich topic in mathematics. Besides being interesting in its own right, random matrices play a fundamental role in various areas such as statistics, mathematical physics, combinatorics, theoretical computer science, etc.

In this survey, we focus on problems of a combinatorial nature. This is a continuation of an earlier suvery [86]. Since the publication of [86], the field has been flourishing and there has been significant progress, including the solutions of several major conjectures. New methods have been introduced which enable one to consider problems which seemed impenetrable only few years ago. Thus, an update is definitely in order.

We mostly focus on the following discrete models, noticing that the techniques developed for them usually work for a much wider class of ensembles.

- $M_{n}$ : random matrix of size $n$ whose entries are i.i.d. Rademacher random variables (taking values \pm 1 with probability $1 / 2$ ). In various papers, this is referred as the random sign matrix or Bernoulli matrices.

- $M_{n}^{\text {sym }}$ : random symmetric matrix of size $n$ whose (upper triangular) entries are i.i.d. Rademacher random variables.

- Adjacency matrix of a random graph. This matrix is symmetric and at position $i j$ we write 1 if $i j$ is an edge and zero otherwise.

- Laplacian of a random graph.

Model of random graphs. We consider two models: Erdös-Rényi and random regular graphs. For more information about these models, see $[9,4,89]$.

- (Erdös-Rényi) We denote by $G(n, p)$ a random graph on $n$ vertices, generated by drawing an edge between any two vertices with probability $p$, independently.

- (Random regular graph) A random regular graph on $n$ vertices with degree $d$ is obtained by sampling uniformly over the set of all simple $d$-regular graphs on the vertex set $\{1, \ldots, n\}$. We denote this graph by $G_{n, d}$. 
It is important to notice that the edges of $G_{n, d}$ are not independent. Because of this, this model is usually harder to study, compared to $G(n, p)$.

We denote by $A(n, p)(L(n, p))$ the adjacency (laplacian) matrix of the ErdösRényi random graph $G(n, p)$ and by $A_{n, d}\left(L_{n, d}\right)$ the adjacency (laplacian) matrix of $G_{n, d}$, respectively.

Notation. In the whole paper, we assume that $n$ is large. The asymptotic notation such as $o, O, \Theta$ is used under the assumption that $n \rightarrow \infty$. We write $A \ll B$ if $A=o(B)$. $c$ denotes a universal constant. All logarithms have natural base, if not specified otherwise.

\section{The singular probability}

The most famous combinatorial problem concerning random matrices is perhaps the "singularity" problem. Let $p_{n}$ be the probability that $M_{n}$ is singular. Trivially,

$$
p_{n} \geq 2^{-n}
$$

as the RHS is the probability that the first two rows are equal.

By choosing any two rows (columns) and also replacing equal by equal up to sign, one can have a slightly better lower bound

$$
p_{n} \geq(4-o(1))\left(\begin{array}{l}
n \\
2
\end{array}\right) 2^{-n}=\left(\frac{1}{2}+o(1)\right)^{n} .
$$

The following conjecture is folklore.

Conjecture 2.1 (Singularity, non-symmetric). $p_{n}=\left(\frac{1}{2}+o(1)\right)^{n}$.

One can formulate even more precise conjectures, based on the following belief

Phenomenon I. The dominating reason for singularity of a random matrix is the dependency between a few rows/columns.

For instance, (1) suggests

Conjecture 2.2. $p_{n}=(2+o(1)) n^{2} 2^{-n}$.

Examining the dependence between 3,4, 5 etc rows (columns) would lead to stronger conjectures with smaller error terms; see [5].

It is already non-trivial to prove that $p_{n}=o(1)$. This was first done by Komlós [46] in 1967. Later, Komlós (see [9]) found a new proof which gave quantitative bound $p_{n}=O\left(n^{-1 / 2}\right)$. The first exponential bound is due to Kahn, Komlós, and Szemrédi [45], who proved $p(n) \leq .999^{n}$. Their arguments were simplified by Tao and $\mathrm{Vu}$ in [75], resulting in a slightly better bound $O\left(.958^{n}\right)$. Shortly afterwards, Tao and $\mathrm{Vu}$ [76] combined the approach from [45] with the idea of inverse theorems (see [80, Chapter 7] or [66] for surveys) to obtained a more significant improvement $p(n) \leq(3 / 4+o(1))^{n}$. With an additional twist, Bourgain, $\mathrm{Vu}$, and Wood [13] improved the bound further to $p(n) \leq\left(\frac{1}{\sqrt{2}}+\right.$ 
$o(1))^{n}$. In a different direction, Rudelson and Vershynin reproved KKS result in a stronger form involving a lower bound for the least singular value [71]

The method from $[76,13]$ enables one to deduce bounds on $p(n)$ directly from simple trigonometrical estimates. For instance, the 3/4-bound comes from the fact that

$$
|\cos x| \leq \frac{3}{4}+\frac{1}{4} \cos 2 x
$$

while the $1 / \sqrt{2}$ bound come from

$$
|\cos x|^{2}=\frac{1}{2}+\frac{1}{2} \cos 2 x .
$$

In 2018, Tikhomirov proved Conjecture 2.1 [82].

Theorem 2.3. $p_{n}=\left(\frac{1}{2}+o(1)\right)^{n}$.

Each of the above mentioned papers contain new, highly non-trivial, ideas, but the core of the matter is a phenomenon called anti-concentration. Tikhomirov's proof combines previous ideas with a new, powerful, double counting argument (which he referred to as inversion of randomness). This, via a sophisticated discretization procedure, reduces matters to studying the anticoncentration properties of random walks with random coefficients. This is perhaps the main difference from previous works which considered random walks with deterministic coefficients. The proof in [82] also provided a bound on the least singular value, extending the result from [71].

To conclude this section, let us discuss a classical anti-concentration result. Let $\mathbf{v}=\left\{v_{1}, \ldots, v_{n}\right\}$ be a set of $n$ non-zero real numbers and $\xi_{1}, \ldots, \xi_{n}$ be i.i.d random Rademacher variables. Define $S:=\sum_{i=1}^{n} \xi_{i} v_{i}, p_{\mathbf{V}}(a)=\mathbf{P}(S=a)$, and $p_{\mathbf{V}}=\sup _{a \in \mathbf{Z}} p_{\mathbf{V}}(a)$.

The problem of estimating $p_{\mathbf{V}}$ came from a paper of Littlewood and Offord in the 1940s [55], as a key technical ingredient in their study of real roots of random polynomials. Erdös, improving a result of Littlewood and Offord, proved the following theorem, which we will refer to as the Erdös-Littlewood-Offord small ball inequality; see [66] for more details.

Theorem 2.4. Let $v_{1}, \ldots, v_{n}$ be non-zero numbers and $\xi_{i}$ be i.i.d Rademacher random variables. Then

$$
p_{\mathbf{V}} \leq \frac{\left(\begin{array}{c}
n \\
\lfloor n / 2\rfloor
\end{array}\right)}{2^{n}}=O\left(n^{-1 / 2}\right)
$$

Theorem 2.4 is a classical result in combinatorics and have many non-trivial extensions with far reaching consequences (see [38, 72, 66], [80, Chapter 7] and the references therein).

To give the reader a feeling about how anti-concentration estimates can be useful in estimating $p_{n}$, let us sketch the proof of $p_{n}=o(1)$. We build $M_{n}$ by adding one random row at a time. Assume that the first $n-1$ rows are independent and form a hyperplane with normal vector $\mathbf{v}=\left(v_{1}, \ldots, v_{n}\right)$. Conditioned 
on these rows, the probability that $M_{n}$ is singular is

$$
\mathbf{P}(X \cdot \mathbf{v}=0)=\mathbf{P}\left(\xi_{1} v_{1}+\cdots+\xi_{n} v_{n}=0\right),
$$

where $X=\left(\xi_{1}, \ldots, \xi_{n}\right)$ is the last row. If we can prove that many of the $v_{i}$ are non-zero, then Theorem 2.4 can be used to finish the job. In order to obtain strong quantitative bounds on $p_{n}$, the following phenomenon proves useful

Phenomenon II. If $\mathbf{P}(X \cdot \mathbf{v}=0)$ is relatively large, then the coefficients $v_{1}, \ldots, v_{n}$ posses a strong additive structure.

For more discussion on this phenomenon and anti-concentration in general, we refer to the survey [66].

Remark 2.5. While polishing this survey, I learned of two new remarkable results (just added to the arxiv). First, Irmatov [43] announced a proof of Conjecture 2.2. His approach seems quite different from all previous ones. Second, Litvak and Tikhomirov [50] announced a solution for a variant of Conjecture 2.2 in the sparse case, for a very wide range of sparsity.

\section{The singular probability: symmetric case}

As an analogue to the problem of the last section, it is natural to raise the question of estimating $p_{n}^{s y m}$, the probability that the symmetric matrix $M_{n}^{s y m}$ singular.

This problem was mentioned to the author by Kalai and Linial (personal conversations) around 2004. To my surprise, at that point, even the analogue of Komlos' 1967 result was not known. According to Kalai and Linial, the following conjecture was circulated by Weiss in the 1980s, although it is quite possible that Komlós had thought about it earlier.

Conjecture 3.1. $p_{n}^{\text {sym }}=o(1)$.

The main difficulty concerning $M_{n}^{s y m}$ is that its rows are no longer independent. This independence plays a critical role in all results discussed in the previous section.

In [23], Costello, Tao, and $\mathrm{Vu}$ found a way to circumvent the dependency. They build the symmetric matrix $M_{n}^{\text {sym }}$ corner to corner. In step $k$, one considers the top left sub matrix of size $k$. The strategy, following an idea of Komlós [46], is to show that with high probability, the co-rank of this matrix, as $k$ increases, behaves like the end point of a biased random walk on non-negative integers which has a strong tendency to go to the left whenever possible. This leads to a confirmation of Weiss' conjecture.

Theorem 3.2. $p_{n}^{s y m}=o(1)$.

The key technical tool in the proof of Theorem 3.2 is the following (quadratic) variant of Theorem 2.4. 
Let us consider the last step in the process when the $(n-1) \times(n-1)$ submatrix $M_{n-1}^{\text {sym }}$ has been built. To obtain $M_{n}^{\text {sym }}$, we add a random row $X=\left(\xi_{1}, \ldots, \xi_{n}\right)$ and its transpose. Conditioning on $M_{n-1}^{s y m}$, the determinant of the resulting $n \times n$ matrix is

$$
\sum_{1 \leq i, j \leq n-1} a_{i j} \xi_{i} \xi_{j}+\operatorname{det} M_{n-1},
$$

where $a_{i j}$ (up to the signs) are the cofactors of $M_{n-1}$. If $M_{n}^{s y m}$ is singular, then its determinant is 0 , which implies

$$
Q:=\sum_{1 \leq i, j \leq n-1} a_{i j} \xi_{i} \xi_{j}=-\operatorname{det} M_{n-1} .
$$

This gives ground for applying an anti-concentration result for quadratic forms.

Motivated by the non-symmetric case, it is natural to conjecture

Conjecture 3.3 (Singularity, symmetric). $p_{n}^{\text {sym }}=(1 / 2+o(1))^{n}$.

We leave it to interested readers to formulate more precise conjectures based on Phenomenon I. The concrete bound from [23] is $n^{-1 / 8}$, which can be easily improved to $n^{-1 / 4}$. Costello [20] improved the bound to $n^{-1 / 2+\epsilon}$ and Nguyen [64] pushed it further to $n^{-\omega(1)}$. Next, Vershynin proved a bound of the form $\exp \left(-n^{c}\right)$, for some small constant $c>0$ [84]. In [29], Ferber and Jain showed that one can take $c=1 / 4$. This was improved further to $c=1 / 2$ by Campos, Mattos, Morris, and Morrison [14].

\section{Ranks and co-ranks}

The singular probability is the probability that the random matrix has corank at least one. What about larger co-ranks? Let us use $p_{n, k}$ to denote the probability that $M_{n}$ has co-rank at least $k$. It is easy to show that

$$
p_{n, k} \geq\left(\frac{1}{2}+o(1)\right)^{k n} .
$$

It is tempting to conjecture that this bound is sharp for constants $k$. In [45], Kahn, Komlós, and Szemerédi showed

Theorem 4.1. There is a function $\epsilon(k)$ tending to zero with $k$ tending to infinity such that

$$
p_{n, k} \leq \epsilon(k)^{n} .
$$

In [13], Bourgain, $\mathrm{Vu}$, and Wood consider a variant of $M_{n}$ where the first $l$ rows are fixed and the next $n-l$ are random. Let $L$ be the submatrix defined by the first $l$ rows and denote the model by $M_{n}(L)$. It is clear that corank $M_{n}(L) \geq$ corank $L$. The authors of [13] showed ([13, Theorem 1.4])

Theorem 4.2. There is a positive constant c such that

$$
\mathbf{P}\left(\operatorname{corank} M_{n}(L)>\operatorname{corank} L\right) \leq(1-c)^{n} .
$$


Let us go back to the symmetric model $M_{n}^{s y m}$ and view it from this new angle, exploiting a connection to Erdös-Rényi random graph $G(n, 1 / 2)$. One can see that in distribution

$$
M_{n}^{\text {sym }}=2 A(n, 1 / 2)-J_{n},
$$

where $J_{n}$ is the all-one matrix of size $n$. (Here we allow $G(n, 1 / 2)$ to have loops, so the diagonal entries of $A(n, 1 / 2)$ can be one. If we fix all diagonal entries to be zero, the analysis does not change significantly.) Since $J_{n}$ has rank one, it follows from Theorem 3.2 that with probablity $1-o(1), A(n, 1 / 2)$ has corank at most one.

One can reduce the co-rank to zero by a slightly trickier argument. Consider $M_{n+1}^{\text {sym }}$ instead of $M_{n}^{\text {sym }}$ and normalize (flipping the signs of each row and column if necessary) so that its first row and column are all- negative one. Adding this matrix with $J_{n+1}$, we obtain a matrix of the form

$$
\left(\begin{array}{cc}
0 & 0 \\
0 & M_{n}^{\text {sym }}+J_{n}
\end{array}\right)
$$

Thus we conclude

Corollary 4.3. With probability $1-o(1)$, corank $A(n, 1 / 2)=0$.

From the random graph point of view, it is natural to ask if this statement holds for a different density $p$ and if there is a threshold phenomenon; see [4] or [9] for the definition of threshold. It is clear that the adjacency matrix is singular if the density $p$ is very small. Indeed, if $p<(1-\epsilon) \log n / n$, for any constant $\epsilon>0$, then by the coupon collector theorem, $G(n, p)$ has, with high probability, isolated vertices, which correspond to zero rows in the adjacency matrix. Costello and $\mathrm{Vu}[21]$ proved that $\log n / n$ is the right threshold.

Theorem 4.4. For any constant $\epsilon>0$, with probability $1-o(1)$,

$$
\operatorname{corank} A(n,(1+\epsilon) \log n / n)=0 \text {. }
$$

Strengthening Theorem 4.4, Basak and Rudelson [8] showed that one can replace $(1+\epsilon) \log n / n$ by $\log n / n+\gamma(n) / n$ where $\gamma(n)$ is any function tending to infinity. In this direction, the most satisfying result is by Addario-Berry and Eslava [1], who proved the following hitting time version. We generate the random graph by adding random edges one by one (the next random edge is uniformly chosen from the set of all available edges). Let $T$ be the first time when the graph has no isolated vertices.

Theorem 4.5. With probability $1-o(1)$, the graph is full rank at time $T$.

For $p<\log n / n$, the co-rank of $A(n, p)$ is no longer zero and its behavior, as a random variable, is not entirely understood. For the case when $p=c \log n / n$ for some constant $0<c<1$, Costello and $\mathrm{Vu}[22]$ showed that with probability $1-o(1)$, the co-rank is determined by small subgraphs whose structure forces the rank to drop, which is consistent with Phenomenon I. For example, 
Theorem 4.6. For any constant $\epsilon>0$ and $(1 / 2+\epsilon) \log n / n<p<(1-$ $\epsilon) \log n / n$, with probability $1-o(1)$, corank $A(n, p)$ equals the number of isolated vertices.

For a smaller $p$, one needs to take into account other small structures such as cherries (a cherry is a pair of vertices of degree one with a common neighbor; in the matrix, this subgraph forces two identical rows). The main result of [22] gives a precise formula for the co-rank in term of these parameters.

When $p=c / n, c>1, G(n, p)$ consists of a giant component and many small components. It makes sense to focus on the giant component which we denote by $\operatorname{Giant}(n, p)$. Since Giant $(n, p)$ has cherries, the adjacency matrix of $\operatorname{Giant}(n, p)$ is singular (with high probability). However, if we look at the $k$-core of $\operatorname{Giant}(n, p)$, for a sufficiently large $k$, it seems plausible that this subgraph has full rank. (A $k$-core of a graph $G$ is a maximal connected subgraph of $G$ in which all vertices have degree at least $k$.)

Conjecture 4.7 (k-core). Let $c>1$ be a constant and set $p=c / n$. There is a constant $k_{0}$ such that for all $k \geq k_{0}$ the following holds. With probability $1-o(1)$, the adjacency matrix of the $k$-core of Giant $(n, p)$ is non-singular.

Bordenave, Lelarge, and Salez [10] proved the following asymptotic result

Theorem 4.8. Consider $G(n, c / n)$ for some constant $c>0$. Then with probability $(1-o(1)) n$,

$$
\operatorname{rank}(A(n, c / n))=\left(2-q-e^{-c q}-c q e^{-c q}+o(1)\right) n,
$$

where $0<q<1$ is the smallest solution of $q=\exp (-c \exp -c q)$.

In [18], Coja-Oghlan, Ergür, Gao, Hetterich, and Rolvien studied random matrices with prescribed number of non-zeroes in each row and column and achieved an asymptotically sharp estimate for the rank; see [18] for details.

\section{Random regular graphs}

Let us consider the random regular graph $G_{n, d}$. For $d=2, G_{n, d}$ is just the union of disjoint circles. It is not hard to show that with probability $1-o(1)$, one of these circles has length divisible by 4 , and thus its adjacency matrix is singular (in fact, its corank is $\Theta(n)$ as the number of circles of length divisible by 4 is of this order). In [85], the author raised the following conjecture (which later appeared in $[35,86]$ as well)

Conjecture 5.1 (Singularity of Random regular graphs). For any $3 \leq d \leq$ $n-1$, with probability $1-o(1) A_{n, d}$ is non-singular.

Many of the earlier works on this conjecture considered the non-symmetric model, namely, random directed regular graphs. In this model, the matrix is chosen uniformly among all (not necessarily symmetric) $(0,1)$ matrices with exactly $d$ ones in each column and row. For this non-symmetric model, Conjecture 5.1 was confirmed for $C \ln ^{2} n \leq d \leq n-C \ln ^{2} n$ by Cook [19], and for 
$C_{n} \leq d \leq n / C \ln ^{2} n$ by Litvak, Lytova, Tikhomirov, Tomczak-Jaegermann, and Youssef [51], where $C$ is a sufficiently large constant and $C_{n}$ tends to infinity arbitrarily slowly; see also [53] for an estimate on the least singular value. Furthermore, Litvak et al. [52] also showed (whp) that the rank is at least $n-1$ for any $d$. Huang showed that the rank equals $n$ (whp) for any fixed $d$; see [40].

For the (original) symmetric case. Landon, Sose, and Yau [49] showed that the conjecture holds for $d \geq n^{c}$ for any constant $c$, as a corollary of a more general and precise universality theorem. The most challenging case, $d$ being a constant, was solved recently by Meszaros [61] and Huang [39]. In particular, Huang proved

Theorem 5.2. For any fixed $d \geq 3$, the probability that $A_{n, d}$ is singular is at most $n^{-c}$ for some constant $c>0$.

Huang's proof showed that one can take $c=\min \{1 / 8,(d-2) /(5 d-6)\}$. On the other hand, he noted that the probability that $A_{n, d}$ is singular is at least $n^{-d+2}$. It is interesting open question to find the sharp value of the exponent.

\section{Determinant and permanent}

Let us start with a basic question

Question 6.1. How big is the determinant of $M_{n}$ ?

This was the original motivation of Komlós' study, which started the line of research discussed in Section 2; see [46, 47]. However, the fact that $M_{n}$ is non-singular (whp) alone does not give any non-trivial estimate on the order of magnitude of $\left|\operatorname{det} M_{n}\right|$.

As all rows of $M_{n}$ has length $\sqrt{n}$, Hadamard's inequality implies that $\left|\operatorname{det} M_{n}\right| \leq n^{n / 2}$. It was conjectured that with probability close to $1,\left|\operatorname{det} M_{n}\right|$ is close to this upper bound.

Conjecture 6.2. Almost surely $\left|\operatorname{det} M_{n}\right|=n^{(1 / 2-o(1)) n}$.

This conjecture is supported by a well-known observation of Turán.

$$
\mathbf{E}\left(\left(\operatorname{det} M_{n}\right)^{2}\right)=n !
$$

To verify this, notice that

$$
\left(\operatorname{det} M_{n}\right)^{2}=\sum_{\pi, \sigma \in S_{n}}(-1)^{\operatorname{sign} \pi+\operatorname{sign} \sigma} \prod_{i=1}^{n} \xi_{i \pi(i)} \xi_{i \sigma(i)} .
$$

By linearity of expectation and the fact that $\mathbf{E}\left(\xi_{i}\right)=0$, we have

$$
\mathbf{E}\left(\operatorname{det} M_{n}\right)^{2}=\sum_{\pi \in S_{n}} 1=n !
$$

It follows immediately by Markov's bound that for any function $\omega(n)$ tending to infinity with $n$,

$$
\left|\operatorname{det} M_{n}\right| \leq \omega(n) \sqrt{n !}
$$

with probability tending to 1 . 
A statement of Girko (the main result of $[37,36]$ ) implies that $\left|\operatorname{det} M_{n}\right|$ is typically close to $\sqrt{n !}$. However, his proof appears to contain some gaps (see [67] for details).

In [75], Tao and $\mathrm{Vu}$ established the matching lower bound, confirming Conjecture 6.2 .

Theorem 6.3. With probability $1-o(1)$,

$$
\left|\operatorname{det} M_{n}\right| \geq \sqrt{n !} \exp (-29 \sqrt{n \log n}) .
$$

We sketch the proof very briefly as it contains a useful lemma.

First view $\left|\operatorname{det} M_{n}\right|$ as the volume of the parallelepiped spanned by $n$ random $\{-1,1\}$ vectors. This volume is the product of the distances from the $(d+1)$ st vector to the subspace spanned by the first $d$ vectors, where $d$ runs from 0 to $n-1$. We are able to obtain a very tight control on this distance (as a random variable), thanks to the following lemma, which can be proved using a powerful concentration inequality by Talagrand [75].

Lemma 6.4. Let $W$ be a fixed subspace of dimension $1 \leq d \leq n-4$ and $X$ a random \pm 1 vector. For any $t>0$

$$
\mathbf{P}(|\operatorname{dist}(X, W)-\sqrt{n-d}| \geq t+1) \leq 4 \exp \left(-t^{2} / 16\right) .
$$

The lemma, however, is not applicable when $d$ is very close to $n$. In this case, we need to make use of the fact that $W$ is random. Lemma 6.4 appears handy in many studies involving high dimensional probability.

Now we turn to the symmetric model $M_{n}^{s y m}$. Again, by Hadamard's inequality $\left|\operatorname{det} M_{n}^{s y m}\right| \leq n^{n / 2}$.

Conjecture 6.5. With probability $1-o(1)$

$$
\left|\operatorname{det} M_{n}^{\text {sym }}\right|=n^{(1 / 2-o(1)) n} .
$$

Turán's identity no longer holds because of a correlation caused by symmetry. However, one can still show

$$
\mathbf{E}\left(\operatorname{det} M_{n}^{\text {sym }}\right)^{2}=n^{(1+o(1)) n} .
$$

On the other hand, proving a lower bound for $\left|\operatorname{det} M_{n}^{\text {sym }}\right|$ was more difficult. Recall that one can interpret the determinant as the product of singular values, which, in this case, are the absolute values of the eigenvalues. By Wigner semi-circle law (see [6]), we know the asymptotic of most of the singular values. However, this law does not say anything about the smallest singular value which, in principle. could be very close to zero. The problem of bounding the least singular value from below was solved by Nguyen [63] and Vershynin [84]. The results by Nguyen and Vershynin, combined with Wigner semi-circle law, confirm Conjecture 6.5.

Theorem 6.6. With probability $1-o(1)$

$$
\left|\operatorname{det} M_{n}^{\text {sym }}\right|=n^{(1 / 2-o(1)) n} .
$$


The interested readers can find more precise results, which write down the limiting law of the determinant in $[37,36,67,74,12]$. Let us now go back to the random regular graphs. Consider the most interesting case when $d$ is a constant, we know that the matrix $A_{n, d}$ (whp) has full rank, so the determinant is non-zero. Its magnitude, however, is unknown.

Question 6.7. Estimate the determinant of $A_{n, d}$. Find the limiting law.

A completely open problem is to bound the probability that $\operatorname{det} M_{n}$ (or other random determinant) takes on a particular non-zero value. In contrast to the singularity conjecture (which addresses the case $\operatorname{det} M_{n}=0$ ), it seems that for any value $x \neq 0, \mathbf{P}\left(\operatorname{det} M_{n}=x\right)$ is sub-exponential. (This was first suggested to the author by Kalai in the early 2000s.) In fact, in view of Turan's identity, we conjecture that

Conjecture 6.8 (Determinant). For any $x \neq 0, \mathbf{P}\left(\operatorname{det} M_{n}=x\right) \leq n^{-(1 / 2+o(1)) n}$.

The best current upper bound is exponential [45]. A much weaker conjecture is that size of the support of $\operatorname{det} M_{n}$ is super-exponential. But even this is not known. The new developments discussed in Section 10 may shed some light on this problem.

Let us now turn to the permanent. Recall the formal definition of the determinant of a matrix $M$ (with entries $m_{i j}, 1 \leq i, j \leq n$ )

$$
\operatorname{det} M:=\sum_{\pi \in S_{n}}(-1)^{\operatorname{sign} \pi} \prod_{i=1}^{n} m_{i \pi(i)} .
$$

The permanent of $M$ is defined as

$$
\text { Per } M:=\sum_{\pi \in S_{n}} \prod_{i=1}^{n} m_{i \pi(i)}
$$

Any question for determinant has its natural analogue for permanent. But typically, the problem becomes much harder as permanent, unlike determinant, does not admit any useful geometric or linear algebraic interpretation. On the other hand, it is easy to see that Turán's identity still holds, namely

$$
\mathbf{E}\left(\operatorname{Per} M_{n}\right)^{2}=n !
$$

It suggests that $\mid$ Per $M_{n} \mid$ is typically $n^{(1 / 2-o(1)) n}$. However, this was much harder to prove than its determinant counterpart. The following conjecture, which is the the permanent analogue of Komlós' 1967 result $p_{n}=o(1)$, was open for several decades

Conjecture 6.9. $\mathbf{P}\left(\operatorname{Per} M_{n}=0\right)=o(1)$.

In 2007, Tao and $\mathrm{Vu}$ [78] found an entirely combinatorial approach to treat the permanent problem, relying on the formal definition (5) and making heavy use of martingale techniques from probabilistic combinatorics. They proved 
Theorem 6.10. With probability $1-o(1)$

$$
\left|\operatorname{Per} M_{n}\right|=n^{(1 / 2-o(1)) n} .
$$

As far as order of magnitude is concerned, the still missing piece of the picture is the symmetric counterpart of Theorem 6.10.

Conjecture 6.11. With probability $1-o(1)$

$$
\left|\operatorname{Per} M_{n}^{\text {sym }}\right|=n^{(1 / 2-o(1)) n} .
$$

Motivated by the singularity problem, it is of interest to find a strong estimate for the probability that the permanent is zero. In this aspect, we believe that determinant and permanent behave differently and conjecture

Conjecture 6.12 (Permanent). The probability that Per $M_{n}=0$ is super exponentially small in $n$.

The current bound is polynomial in $n$ [78]. We do not know anything about the distribution of $\left|\operatorname{Per} M_{n}\right|$, either. Even simulation is challenging, as computing permanent is a well known \#P-complete problem; see [83].

\section{Graph expansion and the second eigenvalue}

Let $G$ be a connected graph on $n$ points and $A$ its adjacency matrix with eigenvalues $\lambda_{1} \geq \lambda_{2} \geq \cdots \geq \lambda_{n}$. If $G$ is $d$-regular then $\lambda_{1}=d$ and by PerronFrobenius theorem no other eigenvalue has larger absolute value. A parameter of fundamental interest is

$$
\lambda(G):=\max _{\left|\lambda_{i}\right|<d}\left|\lambda_{i}\right| .
$$

One can derive interesting properties of the graph from the value this parameter. The general phenomenon here is

Phenomenon III. If $\lambda(G)$ is significantly less than $d$, then the edges of $G$ distribute like in a random graph with edge density $d / n$.

A representative result is the following [4]. Let $A, B$ be sets of vertices and $E(A, B)$ the number of edges with one end point in $A$ and the other in $B$, then

$$
\left|E(A, B)-\frac{d}{n}\right| A|| B|| \leq \lambda(G) \sqrt{|A||B|} .
$$

Notice that the term $\frac{d}{n}|A||B|$ is the expectation of the number of edges between $A$ and $B$ if $G$ is random (in the Erdös-Rényi sense) with edge density $d / n$. Graphs with small $\lambda$ are often called pseudo-random $[4,15,48]$.

One can use this information about edge distribution to derive various properties of the graph (see [48] for many results of this kind). The whole concept 
can be generalized for non-regular graphs, using the Laplacian rather than the adjacency matrix (see, for example, [16]).

From (6), it is clear that the smaller $\lambda$, the more "random" is G. But how small can $\lambda$ be?

In what follows, we restrict ourselves to the most interesting case when $d$ is fixed and $n$ tends to infinity. In this case, Alon and Boppana (see [3]) proved that

$$
\lambda(G) \geq 2 \sqrt{d-1}-o(1)
$$

Graphs which satisfy $\lambda(G)<2 \sqrt{d-1}$ are called Ramanujan graphs. It is very hard to construct such graphs explicitly, and all known constructions, such as those by Lubotzky, Phillip, and Sarnak [54] and Margulis [60] rely heavily on number theoretic results, which, in turn, requires $d$ to have specific values. A more combinatorial approach was found few years ago by Markus, Spielman, and Snivastava [59]. Their method (at least in the bipartite case) works for all $d$, but the construction is not explicit.

Theorem 7.1. A bipartite Ramanujan graph exists for all fixed degrees $d \geq 3$ and sufficiently large $n$.

While showing the existence of Ramanujan graphs is already highly nontrivial, the real question, in our opinion, is to compute the limiting distribution of $\lambda\left(G_{n, d}\right)$, which would lead to the exact probability of a random regular graph being Ramanujan; see [62] for a discussion and some numerical simulation.

A weaker conjecture, by Alon [3], asserts that for any fixed $d$, with probability $1-o(1)$

$$
\lambda_{2}\left(G_{n, d}\right)=2 \sqrt{d-1}+o(1) .
$$

Friedman [32] and Kahn and Szemerédi [44] showed that if $d$ is fixed and $n$ tends to infinity, then with probability $1-o(1), \lambda\left(G_{n, d}\right)=O(\sqrt{d})$. Friedman, in a highly technical paper [33], used the moment method to prove Alon's conjecture (see also [34] for a recent generalization)

Theorem 7.2. For any fixed $d$ and $n$ tending to infinity, with probability $1-o(1)$

$$
\lambda\left(G_{n, d}\right)=2 \sqrt{d-1}+o(1)
$$

For more recent developments concerning Friedman's theorem, including a new, shorter proof by Bordenave, see $[70,11]$.

\section{Simple spectrum}

A matrix has simple spectrum if its eigenvalues are different. We discuss the following basic question

Question 8.1. Are random matrices simple?

It is easy to see that if the entries have continuous distribution, then the spectrum is simple with probability 1 . On the other hand, the discrete case is far from trivial. In particular, Babai raised the following conjecture in the 1980s: 
Conjecture 8.2. With probability $1-o(1), G(n, 1 / 2)$ has a simple spectrum.

The motivation came from the well-known result (proved by Leighton-Miller and Babai-Grigoriev-Mount; see [7]) that the notorious graph isomorphism problem is in $\mathbf{P}$ within the class of graphs with simple spectrum. Few years ago, Tao and $\mathrm{Vu}[81]$ proved this conjecture.

Theorem 8.3 (Simple Spectrum). Babai's conjecture holds.

The same proof applies for $M_{n}^{\text {sym }}$ (and many other ensembles). Let $s_{n}$ be the probability that the spectrum of $M_{n}^{s y m}$ is not simple. We observe that $s_{n} \geq 4^{-n}$, which is the probability that the first 3 rows are the same (which guarantees that zero is an eigenvalue with multiplicity at least 2 ). We conjecture

Conjecture 8.4 (Simplicity). $s_{n}=(4+o(1))^{-n}$.

The current best upper bound is $s_{n} \leq e^{-n^{c}}$ for some small constant $c>0$ [65]. Let us now formulate the singular value version of Babai's conjecture.

Conjecture 8.5. With probability $1-o(1)$, the singular values of $M_{n}^{\text {sym }}$ are different.

Notice that the singular values of a symmetric matrix are the absolute values of its eigenvalues. Thus, this conjecture asserts that there is no two eigenvalues adding up to zero.

One can pose the same questions for $M_{n}$. In this direction, Ge [42] proved the analogue of Theorem 8.3, showing that with probability $1-o(1)$, the spectrum of $M_{n}$ is simple. In a very recent paper, Luh and O'rourke [56] proved the first exponential bound, showing that the probability that the spectrum of $M_{n}$ is not simple is at most $2^{-c n}$, for some constant $c>0$. It looks plausible that Conjecture 8.4 holds for $M_{n}$ as well. The $M_{n}$ analogue of Conjecture 8.5 is also open.

\section{Normality}

Another basic notion in linear algebra is that of normality. An $n \times n$ real matrix $A$ normal if $A A^{T}=A^{T} A$. Few years ago, the author raised the following question.

Question 9.1. How often is a random matrix normal?

Despite the central role of normal matrices in matrix theory, to our surprise, we found no previous results concerning this question. We consider $M_{n}$ and denote by $\nu_{n}$ the probability that $M_{n}$ is normal. Clearly, the probability that $M_{n}$ is symmetric is $2^{-(0.5+o(1)) n^{2}}$. Since symmetric matrices are normal,

$$
\nu_{n} \geq 2^{-(0.5+o(1)) n^{2}} .
$$

We conjecture that this lower bound is sharp.

Conjecture 9.2 (Normality).

$$
\nu_{n}=2^{-(0.5+o(1)) n^{2}} .
$$


In [26], Deneanu and Vu proved

Theorem 9.3.

$$
\nu_{n} \leq 2^{-(0.302+o(1)) n^{2}}
$$

Notice that in previous sections, the conjectural bounds are often of the form $2^{-(c+o(1)) n}$, for some constant $c>0$. While this probability is small, it is still much larger than $2^{-\Omega\left(n^{2}\right)}$, which enables one to exclude very rare events (those occurring with probability $2^{-\omega(n)}$ ) and then condition on their complement.

The difficulty with the normality problem is that we are aiming at a bound which is extremely small (notice that any non-trivial event concerning $M_{n}$ holds with probability at least $2^{-n^{2}}$, which is the mass of a single \pm 1 matrix). There is simply no non-trivial event of probability $1-2^{-\omega\left(n^{2}\right)}$ to condition on. Key to [26] is a new observation that for any given matrix, we can permute its rows and columns so that the ranks of certain submatrices follow a given pattern. The fact that there are only $n !=2^{o\left(n^{2}\right)}$ permutations works in our favor and enables us to execute a different type of conditioning.

Ferber, Jain, and Zhao [30] noticed that one lemma in [26] can be improved, and reworking the whole argument one could improve the constant .302 slightly (maybe at the 5 th decimal place).

Another problem where $2^{-(.5+o(1)) n^{2}}$ could be the right answer is bounding the probability that all eigenvalues of $M_{n}^{\text {sym }}$ are integers (they are apparently real).

Conjecture 9.4 (Integral spectrum). The probability that $M_{n}^{\text {sym }}$ has an integral spectrum is $2^{-(.5+o(1)) n^{2}}$.

In [2], Ahmadi, Alon, Blake, and Shparlinski showed that the probability that $A(n, 1 / 2)$ has an integral spectrum is at most $2^{-n / 400}$. Costello and Williams [24] improved this bound to $2^{-c n^{3 / 2}}$, for some constant $c>0$. Their proof can be modified to yield the same result for $M_{n}^{s y m}$. We also conjecture the $M_{n}$ analogue of the above conjecture to hold

Conjecture 9.5 (Gaussian integral spectrum). The probability that all eigenvalues of $M_{n}$ are gaussian integers is $2^{-(1+o(1)) n^{2}}$.

\section{Sandpile groups of random graphs}

Given a graph $G$, its Laplacian is defined as

$$
L(G)=A(G)-D(G)
$$

where $A(G)$ is the adjacency matrix and $D(G)$ is a diagonal matrix whose $i$ th entry is the degree of the $i$ th vertex. If $G$ is $d$-regular, then $L(G)=A(G)-d I$.

Let $Z$ be the set of integer vectors in $\mathbf{R}^{n}$ whose coordinates sum up to zero. It is clear that the row vectors of $L(G)$ is a subset of $Z$. Let $R$ be the abelian group consisting of integer linear combinations of these vectors. The group $S:=Z / R$ is called the sand pile group of $G$. It is known that $|S|$ equals the product of the 
non-zero eigenvalues of $L(G)$, which equals the number of spanning trees of $G$ (Kirkhoff's theorem).

In [87], Wood studied the structure of $S$, when $G=G(n, p)$ for any fixed $p$ 1 . First, it is shown that for any fixed finite abelian group $H$

$$
\mathbf{P}(S=H)=o(1) .
$$

A finer question is the following. Any finite abelian group $H$ is the direct product of its Sylow subgroups. Now fix a prime $\mathbf{p}$ and a $\mathbf{p}$-group $H$. What is the chance that the $\mathbf{p}$-Sylow subgroup of $S$ equals $H$ ? Wood [87] proved that this probability is asymptotically

$$
\frac{f(H)}{|H||A u t(H)|} \prod_{j=0}^{\infty}\left(1-\mathbf{p}^{-2 j-1}\right)
$$

where $f(H)$ is the number of bilinear, symmetric, perfect maps from $H \times H$ to $\mathbf{C}^{*}$. (For a concrete formula for $f(H)$, see [87].) Wood noted that this is similar (but not quite the same) to a formula suggested by Cohen-Lenstra heuristics.

If $|S|=|\operatorname{det} L(G)|$ is divisible by $\mathbf{p}$, then its $\mathbf{p}$-Sylow subgroup must be non-trivial. Thus, one can use the result to compute the probability that $|S|$ is divisible by $\mathbf{p}$.

Corollary 10.1. Let $\mathbf{p}(n, p)$ be the probability that $\operatorname{det} L(n, p))$ is divisible by p, then

$$
\mathbf{p}(n, p)=(1+o(1))\left(1-\prod_{j \geq 0}\left(1-\mathbf{p}^{-2 j-1}\right)\right) .
$$

For instance, the probability that the number of spanning trees of $G(n, p)$ is even is $\approx .5806 \ldots$. Interestingly, this probability does not depend on the density $p$, as long as it is fixed. In [61], Meszaros extended Wood's theorem to random regular graphs, and used this result to prove the non-singularity of random regular graphs with fixed degrees. See also [88, 17, 57, 58] for related results in this direction.

Let us go back to $M_{n}$, which defines a map from $\mathbf{Z}^{n}$ to itself. As shown in Section 2, this map is (whp) injective. But how often is it surjective? Notice that $M_{n}$ is surjective iff $\left|\operatorname{det} M_{n}\right|=1$, thus the probability of being surjective tends to zero with $n$ as discussed in Section 6.

From this point of view, a recent result of Nguyen and Wood [68] is quite surprising. Consider a $n \times(n+1)$ random matrix with iid Rademacher entries. This matrix defines a map from $\mathbf{Z}^{n+1}$ to $\mathbf{Z}^{n}$. What is the probability that this map is surjective? Nguyen and Wood showed that this probability is

$$
(1+o(1)) \prod_{k \geq 2} \zeta(k)^{-1} \approx .4358
$$

\footnotetext{
${ }^{1}$ The Woods in this section and Section 2 are M. M. Wood and P. M. Wood, respectively.
} 
which is bounded away from both 0 and 1 . (Here $\zeta$ is the zeta function.) An important step in the proof shows that with respect to different primes $\mathbf{p}_{1}, \ldots, \mathbf{p}_{k}$, the distributions of a random determinant $\bmod \mathbf{p}_{i}$ are approximately independent.

\section{Miscellany}

An interesting (and seemingly hard) conjecture is the following, which came up in the conversation between the author and P. M. Wood in 2009. Later, Babai informed us that he made the same conjecture (unpublished) in the 1970s.

Conjecture 11.1 (Irreducibility). With probability $1-o(1)$, the characteristic polynomial of $M_{n}$ is irreducible.

In [86], the author raised the following conjecture, which asserts that spectra can be used as finger prints.

Conjecture 11.2 (Finger Print). A \pm 1 matrix is determined by its spectrum if no other \pm 1 matrices (not counting trivial permutations) have the same spectrum. Then almost all \pm 1 matrices are determined by their spectrum.

One can raise the same question for $M_{n}^{s y m}$ or $G(n, 1 / 2)$. It is known that there are non-isomorphic co-spectral graphs. However, these should form a negligible part of the set of all graphs.

The matrix $M_{n}$ is (whp) non-symmetric. Thus, there is no obvious reason for its to have many real eigenvalues. (The oddity of $n$ would guarantee one real eigenvalue, but nothing more.) The following conjecture is motivated by our joint work with Tao in [79].

Conjecture 11.3 (Real Eigenvalues). $M_{n}$ has, with high probability, $\Theta(\sqrt{n})$ real eigenvalues.

Edelman, Kostlan, and Shub [27] obtained a formula for the expectation of the number of real eigenvalues for a gaussian matrix (which is or order $\Theta(\sqrt{n})$ ). In [79], Tao and $\mathrm{Vu}$ proved that the same formula holds (in the asymptotic sense) for certain random matrices with entries $(0, \pm 1)$. However, we do not know anything about $M_{n}$. As a matter of fact, even the following "first step" seems hard

Problem 11.4 (Two real roots). Prove that $M_{n}$ has, with high probability, at least 2 real eigenvalues.

The next problem bears some resemblance to the famous "rigidity" problem in computer science. Given a \pm 1 square matrix $M$, we denote by $\operatorname{Res}(M)$ the minimum number of entries we need to switch (from 1 to -1 and vice versa) in order to make $M$ singular. Thus, Res can be seen as the resilience of the matrix against an effort to reduce its rank. It is easy to show that $\operatorname{Res}\left(M_{n}\right)$ is, with high probability, at most $(1 / 2+o(1)) n$. 
Conjecture 11.5 (Rank Resilience). With probability $1-o(1), \operatorname{Res}\left(M_{n}\right)=$ $(1 / 2+o(1)) n$.

For a recent partial result, see [31]. A closely related question (motivated by the notion of local resilience from [73]) is the following. Call a $\{-1,1\}$ ( $n$ by $n$ ) matrix $M$ stubborn if all matrices obtained by switching (from 1 to -1 and vice versa) the diagonal entries of $M$ are non-singular (there are $2^{n}$ such matrices).

Conjecture 11.6 (Local resilience). With probability $1-o(1), M_{n}$ is stubborn.

We do not discuss eigenvectors here, the interested reader may want to consult [69] for a survey. See also $[25,41]$ for recent results on nodal domains of random graphs.

\section{Acknowledgments}

The author would like to thank NSF and AFORS for their generous support and K. Luh, C. Koenig, L. Addario-Berry, H. Nguyen, J. Huang, A. Litvak, K. Tikhomirov, and the referees for useful comments.

\section{References}

[1] L. Addario-Berry and L. Eslava, Hitting time theorems for random matrices, Combinatorics, Probability and Computing 23 (2014), 635-669. MR3249218

[2] O. Ahmadi, N. Alon, I. Blake, and I. Shparlinski, Graphs with integral spectrum, Linear Algebra and its Applications 430 (2009), 547-552. MR2460536

[3] N. Alon, Eigenvalues and expanders, Combinatorica 6 (1986), no. 2, 83-96. MR0875835

[4] N. Alon and J. Spencer, The probabilistic method, 3rd ed., John Wiley 86 Sons Inc., Hoboken, NJ, 2008. MR2437651

[5] R. Arratia and S. DeSalvo, On the singularity of random Bernoulli matrices: Novel integer partitions and lower bound expansions, Ann. Comb. 17 (2013), no. 2, 251-274. MR3056767

[6] Z. Bai and J. Silverstein, Spectral analysis of large dimensional random matrices. Second edition. Springer Series in Statistics. Springer, New York, 2010. MR2567175

[7] L. Babai, D. Grigoryev, and D. Mount, Isomorphism of graphs with bounded eigenvalue multiplicity, In: Proceedings of the 14th Annual ACM Symposium on Theory of Computing, 310-324 (1982).

[8] A. Basak and M. Rudelson, Sharp transition of the invertibility of the adjacency matrices of sparse random graphs, arXiv:1809.08454. MR3620692

[9] B. Bollobás, Random graphs. Second edition, Cambridge Studies in Advanced Mathematics, 73. Cambridge University Press, Cambridge, 2001. MR1864966

[10] C. Bordenave, M. Lelarge, and J. Salez, The rank of diluted random graphs, Ann. Probab. 39 (2011), no. 3, 1097-1121. MR2789584 
[11] C. Bordenave, A new proof of Friedman's second eigenvalue Theorem and its extension to random lifts. To appear in Annales Scientifiques de l'Ecole Normale Supérieure, arxiv:1502.04482. MR4203039

[12] P. Bourgade and K. Mudy, Gaussian fluctuations of the determinant of Wigner Matrices, Electronic Journal of Probability 24 (2019), no. 96. MR4017114

[13] J. Bourgain, V. Vu and P. M. Wood, On the singularity probability of discrete random matrices, J. Funct. Anal. 258 (2010), no. 2, 559-603. MR2557947

[14] M. Campos, L. Mattos, R. Morris, and N. Morrison, On the singularity of random symmetric matrices, arXiv:1904.11478.

[15] F. Chung, R. Graham, and R. Wilson, Quasi-random graphs. Combinatorica 9 (1989), no. 4, 345-362. MR1054011

[16] F. Chung, Spectral graph theory, CBMS Series, no. 92 (1997). MR1421568

[17] J. Clancy, T. Leake, N. Kaplan, S. Payne, and M. Matchett Wood, On a Cohen-Lenstra heuristic for Jacobians of random graphs, J. Algebraic Combin. 42 (2015), no. 3, 701-723. MR3403177

[18] A. Coja-Oghlan, A. Ergür, P. Gao, S. Hetterich, and M. Rolvien, The rank of sparse random matrices, arXiv:1906.05757.

[19] N. Cook. On the singularity of adjacency matrices for random regular digraphs, Probab. Theory Related Fields 167 (2017) 143-200. MR3602844

[20] K. Costello, Bilinear and quadratic variants on the Littlewood-Offord problem, Israel J. Math. 194 (2013), no. 1, 359-394. MR3047075

[21] K. Costello and V. Vu, The ranks of random graphs. Random Structures and Algorithm. 33 (2008), 269-285. MR2446482

[22] K. Costello and V. Vu, The rank of sparse random matrices, Combin. Probab. Comput. 19 (2010), no. 3, 321-342. MR2607371

[23] K. Costello, T. Tao, and V. Vu, Random symmetric matrices are almost surely singular, Duke Math. J. 135 (2006), no. 2, 395-413. MR2267289

[24] K. Costello and P. Williams, On the number of integral graphs, Linear Algebra and its Applications 493 (2016), 447-454. MR3452749

[25] Y. Dekel, J. R. Lee, and N. Linial, Eigenvectors of random graphs: Nodal domains, Random Structures and Algorithms 39 (2011), no. 1, 39-58. MR2839984

[26] A. Deneanu and V. Vu, Random matrices: Probability of normality, Advances of Mathematics 346 (2019), 887-907. MR3914182

[27] A. Edelman, E. Kostlan, and M. Shub, How many eigenvalues of a random matrix are real? J. Amer. Math. Soc. 7 (1994), no. 1, 247-267. MR1231689

[28] P. Erdös, On a lemma of Littlewood and Offord, Bull. Amer. Math. Soc. 51 (1945), 898-902. MR0014608

[29] A. Ferber and V. Jain, Singularity of random symmetric matrices: a combinatorial approach to improved bounds, Forum of Mathematics, Sigma, accepted for publication. MR3993806

[30] A. Ferber, V. Jain, and Y. Zhao, On the number of Hadamard matrices via anti-concentration, arXiv:1808.07222.

[31] A. Ferber, K. Luh, and G. McKinley, Resilience of the rank of random 
matrices, arXiv:1910.03619. MR4225782

[32] J. Friedman. On the second eigenvalue and random walks in random dregular graphs. Technical Report CX-TR-172-88, Princeton University, August 1988.

[33] J. Fiedman, A proof of Alon's second eigenvalue conjecture and related problems, Mem. Amer. Math. Soc. 195 (2008), no. 910, viii+100 pp. MR2437174

[34] J. Friedman and D. E. Kohler, The relativized second eigenvalue conjecture of Alon, arXiv:1403.3462.

[35] A. Frieze, Random structures and algorithms. In: Proceedings of the International Congress of Mathematicians, Seoul 2014. Vol. 1, pages 311-340. Kyung Moon Sa, Seoul, 2014. MR3728474

[36] V. L. Girko, A refinement of the central limit theorem for random determinants. (Russian) Teor. Veroyatnost. i Primenen. 42 (1997), no. 1, 6373; translation in Theory Probab. Appl. 42 (1997), no. 1, 121-129 (1998). MR1453330

[37] V. L. Girko, A central limit theorem for random determinants. Teor. Veroyatnost. i Mat. Statist. 21 (1979), 35-39, 164. MR0550240

[38] G. Halász, Estimates for the concentration function of combinatorial number theory and probability, Period. Math. Hungar. 8 (1977), no. 3-4, 197211. MR0494478

[39] J. Huang, Invertibility of adjacency matrices for random d-regular graphs, arXiv:1807.06465 (2018).

[40] J. Huang, Invertibility of adjacency matrices for random d-regular directed graphs, arXiv:1806.01382 (2018).

[41] H. Huang and M. Rudelson, Size of nodal domains of the eigenvectors of a $G(n, p)$ graph, arXiv:1905.00447. MR4129726

[42] S. Ge, PhD Thesis, UCLA 2017, https://escholarship.org/content/ qt1n54260.

[43] A. Irmatov, Singularity of \pm 1 -matrices and asymptotics of the number of threshold functions, arXiv:2004.03400. MR1266256

[44] J. Kahn and E. Szemerédi, STOC 1989.

[45] J. Kahn, J. Komlós, and E. Szemerédi, On the probability that a random \pm 1 matrix is singular, J. Amer. Math. Soc. 8 (1995), 223-240. MR1260107

[46] J. Komlós, On the determinant of $(0,1)$ matrices, Studia Sci. Math. Hungar. 2 (1967), 7-22. MR0221962

[47] J. Komlós, On the determinant of random matrices,Studia Sci. Math. Hungar. 3 (1968), 387-399. MR0238371

[48] M. Krivelevich and B. Sudakov, Pseudo-random graphs. In: More Sets, Graphs and Numbers, 199-262, Bolyai Soc. Math. Stud., vol. 15, Springer, Berlin, 2006. MR2223394

[49] B. Landon, P. Sosoe, and H.-T. Yau, Fixed energy universality of Dyson Brownian motion, arXiv:1609.09011 (2016). MR3914908

[50] A. Litvalk and K. Tikhomirov, Singularity of random sparse Bernoulli matrices, arXiv:2004.03131. MR4076632

[51] A. E. Litvak, A. Lytova, K. Tikhomirov, N. Tomczak-Jaegermann, and 
P. Youssef, Adjacency matrices of random digraphs: singularity and anticoncentration, J. Math. Anal. Appl. 445 (2017), 1447-1491. MR3545253

[52] A. E. Litvak, A. Lytova, K. Tikhomirov, N. Tomczak-Jaegermann, and P. Youssef, The rank of random regular digraphs of constant degree, Journal of Complexity 48 (2018), 103-110. MR3828839

[53] A. E. Litvak, A. Lytova, K. Tikhomirov, N. Tomczak-Jaegermann, and P. Youssef, The smallest singular value of a shifted $d$-regular random square matrix, Prob. Th. Rel. Fields 173 (2019), 1301-1347. MR3936157

[54] A. Lubotzky, R. Phillips, and P. Sarnak, Ramanujan graphs, Combinatorica 8(3) (1988), 261-277. MR0963118

[55] J. E. Littlewood and A. C. Offord, On the number of real roots of a random algebraic equation. III, Rec. Math. [Mat. Sbornik] N.S. 12 (1943), 277-286. MR0009656

[56] K. Luh and S. O'Rourke, Eigenvectors and controllability of non-Hermitian random matrices and directed graphs, arXiv:2004.10543.

[57] K. Maples, Singularity of random matrices over finite fields, arXiv:1012.2372 (2010).

[58] K. Maples, Cokernels of random matrices satisfy the Cohen-Lenstra heuristics, arXiv:1301.1239 (2013).

[59] A. Marcus, D. Spielman, and N. Srivastava, Interlacing families I: bipartite Ramanujan graphs of all degrees, Ann. of Math. 182 (2015), no. 1, 307-325. MR3374962

[60] G. A. Margulis, Explicit group-theoretical constructions of combinatorial schemes and their application to the design of expanders and superconcentrators, Problemy Peredachi Informatsii 24 (1988), 51-60 [in Russian]. MR0939574

[61] A. Mészáros. The distribution of sandpile groups of random regular graphs, arXiv:1806.03736 (2018). MR4155185

[62] S. Miller, T. Novikoff, and A. Sabelli, The distribution of the largest non-trivial eigenvalues in families of random regular graphs, arXiv:math/0611649. MR2433888

[63] H. Nguyen, On the least singular value of random symmetric matrices, Electron. J. Probab. 17 (2012), no. 53. MR2955045

[64] H. Nguyen, Inverse Littlewood-Offord problems and the singularity of random symmetric matrices, Duke Math. J. 161 (2012), no. 4, 545-586. MR2891529

[65] H. Nguyen, T. Tao, and V. Vu, Random matrices: tail bounds for gaps between eigenvalues, Probability Theory and Related Fields 167 (2017), no. 3, 777-816. MR3627428

[66] H. Nguyen and V. Vu, Small probability, inverse theorems, and applications, In: Erdos' 100th Anniversary Proceeding, Bolyai Society Mathematical Studies, vol. 25 (2013). MR3203607

[67] H. Nguyen and V. Vu, Random matrices: Law of the determinant, Annals of Probability 42 (2014), no. 1, 146-167. MR3161483 
[68] H. Nguyen and M. M. Wood, Random integral matrices: universality of surjectivity and the cokernel, https://people.math.osu.edu/nguyen.1261/ cikk/CL-Laplacian.pdf.

[69] S. O'Rourke, V. Vu, K. Wang, Eigenvectors of random matrices: A survey, Journal of Combinatorial Theory, Series A 144 (2016), 361-442. MR3534074

[70] D. Puder, Expansion of random graphs: new proofs, new results, Inventiones Mathematicae 201 (2015), 845-908. MR3385636

[71] M. Rudelson and R. Vershynin, The Littlewood-Offord problem and invertibility of random matrices, Adv. Math. 218 (2008), no. 2, 600-633. MR2407948

[72] A. Sárközy and E. Szemerédi, Uber ein Problem von Erdös und Moser, Acta Arithmetica 11 (1965) 205-208. MR0182619

[73] B. Sudakov and V. Vu, Local resilience of graphs, Random Structures Algorithms 33 (2008), no. 4, 409-433. MR2462249

[74] T. Tao and V. Vu, A central limit theorem for the determinant of a Wigner matrix, Adv. Math. 231 (2012), no. 1, 74-101. MR2935384

[75] T. Tao and V. Vu, On random \pm 1 matrices: Singularity Determinant, Random Structures Algorithms 28 (2006), no. 1, 1-23. MR2187480

[76] T. Tao and V. Vu, On the singularity probability of random Bernoulli matrices, J. Amer. Math. Soc. 20 (2007), no. 3, 603-628. MR2291914

[77] T. Tao and V. Vu, Inverse Littlewood-Offord theorems and the condition number of random matrices, Annals of Math. 169 (2009), 595-632. MR2480613

[78] T. Tao and V. Vu, On the permanent of random Bernoulli matrices, Advances in Mathematics 220 (2009), 657-669. MR2483225

[79] T. Tao and V. Vu, Random matrices: universality of local spectral statistics of non-Hermitian matrices, Annals of Probability 43 (2015), no. 2, 782-874. MR3306005

[80] T. Tao and V. Vu, Additive Combinatorics, Cambridge Univ. Press, 2006. MR2289012

[81] T. Tao and V. Vu, Random matrices have simple spectrum, Combinatorica 37 (2017), 539-553. MR3666791

[82] K. Tikhomirov, Singularity of random Bernoulli matrices, Annals of Mathematics 191 (2020), 593-634. MR4076632

[83] L. Valiant, The complexity of computing the permanent, Theoretical Computer Science 8 (1979), no. 2, 189-201. MR0526203

[84] R. Vershynin, Invertibility of symmetric random matrices, Random Structures and Algorithms 44 (2014), 135-182. MR3158627

[85] V. Vu, Random discrete matrices. In: Horizons of combinatorics, Bolyai Soc. Math. Stud., vol. 17, pages 257-280. Springer, Berlin, 2008. MR2432537

[86] V. Vu, Combinatorial problems in random matrix theory. In: Proceedings of the International Congress of Mathematicians, Seoul 2014, vol. IV, pages 489-508. Kyung Moon Sa, Seoul, 2014. MR3727622

[87] M. M. Wood, The distribution of sandpile groups of random graphs, Journal of the A.M.S. 30 (2017), 915-958. MR3671933 
[88] M. M. Wood, Random integral matrices and the Cohen-Lenstra Heuristics, to appear in American Journal of Mathematics, arXiv:1504.04391. MR3928040

[89] N. C. Wormald, Models of random regular graphs. In: Surveys in Combinatorics, 1999, J. D. Lamb and D. A. Preece (eds.), pages 239-298. MR1725006 